\title{
EFETIVIDADE DO GERENCIAMENTO DE RISCOS CLÍNICOS NA TERAPIA INTENSIVA
}

\author{
Ruy de Almeida Barcellos ${ }^{1}$, Melise Toscan², Ana Cristina Pretto Báo ${ }^{3}$, Simone Coelho Amestoy ${ }^{4}$
}

\begin{abstract}
RESUMO: Objetivou-se mensurar a taxa de efetividade do gerenciamento de riscos clínicos na terapia intensiva. Trata-se de um estudo transversal, com uma abordagem quantitativa, realizado em um hospital filantrópico do município de Caxias do Sul. A amostra foi composta pelos pacientes internados na terapia intensiva inclusos no formulário de gerenciamento de riscos no período de janeiro de 2013 a dezembro de 2014. Os riscos que foram analisados nesta pesquisa foram: queda; lesão de pele; extubação acidental; perda acidental de sonda nasoentérica e sonda nasogástrica; e perda acidental de cateter venoso central. O total da amostra foi de 51220 pacientes-dia. Os resultados encontrados evidenciaram que a taxa de efetividade nos riscos pesquisados, em sua maioria, permaneceu acima de $99 \%$. Conclui-se que a enfermagem tem papel fundamental no gerenciamento de riscos, atividade essa que exige do enfermeiro aperfeiçoamento constante, com foco na qualidade e segurança da assistência.
\end{abstract}

DESCRITORES: Enfermagem; Gestão de Riscos; Unidade de Terapia Intensiva.

\section{EFFECTIVENESS OF CLINICAL RISK MANAGEMENT IN INTENSIVE CARE}

\begin{abstract}
The present study aimed to measure the effectiveness of clinical risk management in intensive care. Cross-sectional study with quantitative approach conducted in a philanthropic hospital in the city of Caxias do Sul. The sample was composed of patients admitted to the intensive care unit included in the risk management assessment form in the period of January 2013 to December 2014. The risks analyzed in this study were fall; skin lesion; unplanned extubation; accidental removal of nasoenteral feeding tube and nasogastric tube and accidental catheter removal for central venous catheter. The sample was composed of 51,220 patients-day. According to the results obtained, the effectiveness of the management of the risks investigated in this study was above $99 \%$. It is concluded that nursing plays a key role in risk management, an activity that requires continuing training of nurses, with focus on care quality and safety.
\end{abstract}

DESCRIPTORS: Nursing; Risk Management; Intensive Care Unit.

\section{EFECTIVIDAD DE LA GESTIÓN DE RIESGOS CLÍNICOS EN LA TERAPIA INTENSIVA}

RESUMEN: Fue la finalidad de este estudio mensurar la taja de efectividad de la gestión de riesgos clínicos en la terapia intensiva. Trasversal y con abordaje cuantitativo, el estudio fue realizado en un hospital filantrópico del municipio de Caxias do Sul. La muestra fue compuesta por los pacientes internados en la terapia intensiva inclusos en el formulario de gestión de riesgos en el periodo de enero de 2013 a diciembre de 2014. Los riesgos analizados en esta investigación fueron caída; lesión de piel; extubación accidental; pérdida accidental de sonda nasoentérica y sonda nasogástrica; y pérdida accidental de catéter venoso central. El total de la muestra fue de 51220 pacientes al día. Los resultados evidenciaron que la taja de efectividad de la mayoría de los riesgos investigados resultó en más de $99 \%$. La conclusión es que la enfermería tiene papel fundamental en la gestión de riesgos, la cual es una actividad que exige al enfermero constante perfeccionamiento, con énfasis en la cualidad y seguridad de la asistencia.

DESCRIPTORES: Enfermería; Gestión de Riesgos; Unidad de Terapia Intensiva.

'Enfermeiro. Doutorando em Ciências da Saúde. Docente de Enfermagem da Faculdade da Serra Gaúcha. Caxias do Sul, RS, Brasil.

${ }^{2}$ Discente de Enfermagem. Faculdade da Serra Gaúcha. Caxias do Sul, RS, Brasil.

${ }^{3}$ Enfermeira. Mestranda em Enfermagem. Universidade Federal de Pelotas. Caxias do Sul, RS, Brasil.

${ }^{4}$ Doutora em Enfermagem. Docente de Enfermagem da Universidade Federal de Pelotas. Pelotas, RS, Brasil.

Autor Correspondente:

Ruy de Almeida Barcellos

Faculdade da Serra Gaúcha

R. Os 18 do Forte, 2366 - 95020-472 - Caxias do Sul, RS, Brasil

E-mail: ruy.barcellos@hotmail.com
Recebido: $15 / 02 / 2016$

Finalizado: $12 / 08 / 2016$ 


\section{INTRODUÇÃO}

As organizações de saúde exercem atividades críticas, em que cada vez mais expõem os pacientes a riscos, o que sujeita ao aparecimento de eventos adversos (EA): incidentes que resultam em dano ao paciente ${ }^{(1)}$. Atualmente, percebe-se a alta incidência destes eventos, tornando-os cada vez mais preocupantes entre profissionais e gestores de saúde, pois problemas deste cunho refletem um marcante distanciamento entre o cuidado real e o cuidado ideal. Diante disso, a segurança do paciente passa a ser essencial para prestar uma assistência de melhor qualidade a seus usuários ${ }^{(2)}$.

A assistência segura tornou-se um assunto das discussões na área da saúde em todo o mundo. O relatório Institute of Medicine, dos Estados Unidos, apresentou estudos que mostram a precária situação de assistência à saúde do país. Os dados revelaram que das 33,6 milhões de internações, 44.000 a 98.000 pacientes, aproximadamente, morreram em consequência de eventos adversos ${ }^{(3)}$.

Diante destes resultados, estimou-se que 180.000 óbitos ocorreram naquele país, em consequência da contribuição destes danos. O relatório do Instituto de Medicina dos Estados Unidos identificou que $7 \%$ dos pacientes que se encontravam internados eram acometidos por erros de medicação. Nas Unidades de Terapia Intensiva (UTI), área complexa e sujeita a diversos riscos, notou-se que $17 \%$ dos pacientes sofriam algum evento adverso importante ${ }^{(3)}$. Diante disso, a Organização Mundial da Saúde (ONU) passou a adotar este tema como prioridade em suas políticas ${ }^{(4)}$.

A partir desta preocupação com a qualidade da assistência ao paciente e com a mitigação dos riscos assistenciais, o gerenciamento de riscos, que envolve a administração desses riscos, pode auxiliar na redução de eventos ao paciente. Esse gerenciamento auxilia os gestores a tomarem decisões dentro da instituição e priorizar ações. Ele faz com que as instituições de saúde adotem como política a prevenção de riscos, com o intuito de criar uma base sólida e segura para a tomada de decisões e para o planejamento da segurança do paciente, mediante o monitoramento de eventos adversos e da diminuição de danos ao paciente, além da prevenção de incidentes ${ }^{(5)}$.

A enfermagem tem papel fundamental no gerenciamento de risco, pois é quem fica maior tempo com a paciente, assim podendo reconhecer potenciais riscos e realizar medidas preventivas para que não se tornem eventos adversos ${ }^{(6)}$.

Com o objetivo de facilitar a organização de um bom gerenciamento de risco, podemos classificar os riscos como clínicos e não-clínicos. O gerenciamento de riscos clínicos tem por objetivo detectar, precocemente, situações que poderiam gerar consequências aos pacientes, à instituição e ao meio ambiente. Ele tem em vista a aplicação de uma série de medidas para prever, identificar e minimizar a ocorrência de eventos que, muitas vezes, causam danos irreversíveis ao paciente ${ }^{(7)}$.

O risco clínico é todo risco associado à ação direta ou indireta do profissional da área da saúde, resultado da deficiência de políticas e ações organizadas na prestação dos cuidados em saúde. Pode-se citar como exemplo de risco clínico: cirurgia não segura; identificação incorreta do paciente; falha na segurança medicamentosa; aquisição de infecção; e risco de queda ${ }^{(8)}$.

O risco não-clínico é aquele que tem relação com a segurança das instalações ou com o atendimento aos processos de prestação de cuidados aos pacientes, além dos riscos gerados da quebra nas condições adequadas de trabalho. Como exemplo dos riscos não-clínicos, tem-se: falta de manutenção de elevadores; proteção radiológica; acidentes ocupacionais; exames periódicos; falta de manutenções preventivas, entre outros ${ }^{(8)}$.

Os riscos de eventos adversos, dos acidentes e das falhas existem em qualquer área de uma instituição de saúde. Dentre essas, destaca-se a UTI, que é um ambiente assistencial de alto risco, onde, muitas vezes, é necessário que o cuidado intensivo seja prestado de forma rápida, evolvendo diversos procedimentos, equipamentos e pessoas, e produzindo uma grande quantidade de informações, devido à gravidade dos pacientes assistidos. Diante disso, pode se tornar uma atividade estressante por estar diretamente ligada a situações de vida ou morte, em que decisões devem ser tomadas de forma rápida. Portanto, essa área exige profissionais capacitados e com aperfeiçoamento, cabendo à enfermagem um papel fundamental para reconhecimento, gerenciamento e prevenção de $\operatorname{riscos}^{(9)}$. 
Em um estudo realizado em um hospital de ensino, foi avaliada a incidência de eventos adversos e incidentes em pacientes de UTI. As taxas de eventos adversos e incidentes por 1.000 pacientes-dia foram, respectivamente, 80,5 e 149,7 sendo $13 \%$ destes graves ou fatais. Do total de pacientes, $20,2 \%$ sofreram ao menos um evento, uma taxa maior que a de estudos não focados em terapia intensiva ${ }^{(2)}$.

Uma pesquisa que abordou o custo financeiro dos eventos adversos em terapia intensiva, revelou que cada evento adverso impõe ao paciente afetado um custo adicional em torno de 4.000,00 dólares e aumenta o tempo de internação em um dia. Esse mesmo estudo apontou um aumento de 31 dias, na média, do tempo de permanência de pacientes que sofreram um evento adverso quando internados em terapia intensiva ${ }^{(10)}$.

Com base no exposto, a questão norteadora do estudo foi: Qual a taxa de efetividade do gerenciamento de riscos clínicos em terapia intensiva? E, como objetivo, mensurar a taxa de efetividade do gerenciamento de riscos clínicos na terapia intensiva.

\section{- MÉTODO}

Trata-se de um estudo transversal com uma abordagem quantitativa. O estudo foi realizado em uma entidade filantrópica, que atende mais de $60 \%$ de seus pacientes pelo Sistema Único de Saúde (SUS), nas unidades de terapia intensiva adulto com o total de 30 leitos.

A amostra do estudo foi composta de paciente inclusos no formulário de gerenciamento de risco diário da instituição, no período de janeiro de 2013 a dezembro de 2014, totalizando 51220 pacientesdia, sendo incluso no formulário apenas pacientes que apresentavam determinado risco.

Os critérios que foram utilizados para inclusão na amostra são: pacientes internados nas unidades de terapia intensiva adulto no período de janeiro de 2013 a dezembro de 2014; e ter sido incluído no formulário de gerenciamento de riscos diário. Os critérios de exclusão utilizados foram: pacientes internados na UTI adulto em um período menor de 24 horas.

Os dados foram obtidos por meio de formulários de gerenciamento de riscos, que são preenchidos diariamente pelo enfermeiro da unidade. Nele, registra-se o número total de pacientes/dia expostos a riscos e o número de pacientes que desenvolveram eventos adversos. Mensalmente, esse formulário é encaminhado ao setor de qualidade da instituição, onde é contabilizado, avaliado e discutido no comitê de gerenciamento de riscos. Ele também é categorizado em riscos clínicos e não-clínicos. Os eventos adversos são organizados em tabelas eletrônicas através do programa Microsoft Excel.

Os riscos analisados nesta pesquisa são: queda; lesão de pele; extubação acidental; perda acidental de sonda nasoentérica (SNE) e sonda nasogástrica (SNG), e perda acidental de cateter venoso central (CVC). Optou-se por avaliar a taxa de efetividade destes riscos devido ao grande número de pacientes/ dia expostos a eles na instituição pesquisada, e por estes serem considerados de gravidade elevada dentro de uma UTI.

Para a coleta de dados, primeiramente foram separados os formulários de gerenciamento das UTIs e, logo após, foi contabilizado o total de cada mês de paciente/dia com o risco, das três UTIs da instituição. Na terceira etapa, foi coletado o número de eventos adversos que ocorrem em cada mês, também nas três unidades. Posteriormente, utilizando estes dados, foi realizado o cálculo para a medição da taxa de efetividade, que representa o valor em percentual da taxa de adesão às barreiras implantadas para prevenção dos riscos. Para realização do cálculo da taxa de efetividade de cada risco, é necessário determinar o número total de pacientes/dia com determinado risco, subtraindo deste o número de eventos adversos ocorridos, ou seja, número de vezes em que ocorreram falhas nas barreiras implementadas para cada risco. Desta forma, para se chegar ao percentual foi aplicada a seguinte fórmula: (número de paciente/dia - eventos adversos ocorridos / número total de paciente/ dia para cada risco x 100).

O estudo foi submetido à apreciação do Comitê de Ética em Pesquisa da instituição em que foi realizada através do parecer número 1.205.253 e aprovada pelo Comitê de Ética em Pesquisa da Faculdade da Serra Gaúcha (FSG) diante do parecer número 1180.964. 
Nos resultados descreve-se o número total de pacientes/dia submetidos ao risco, e o número de eventos notificados. Em seguida, apresenta-se o cálculo da taxa de efetividade dos anos de 2013 e 2014.

Conforme a Tabela 1, no ano de 2013, foram registrados 4.560 pacientes/dia com risco de queda, e foram notificados apenas três casos de queda nas UTIs. No risco de lesão de pele, foram registrados 4.147 pacientes/dia com o risco, e foram notificados seis eventos. Referente ao risco de extubação acidental, 1.528 pacientes/dia foram expostos ao risco e, dentre esses, 18 eventos foram notificados. Referente ao risco de perda acidental de SNE e de SNG, foram registrados 3.362 pacientes/dia submetidos a este risco e 32 foram notificados. No risco de perda acidental de cateter venoso central, foram transcritos 3.788 pacientes/dia com este risco e cinco foram notificados. Estes resultados mostraram que a taxa de efetividade, no ano de 2013, permaneceu acima de $90 \%$ nos riscos que foram explorados.

No ano de 2014, conforme Tabela 2, no risco de queda foram registrados 8.626 pacientes/dia, com quatro notificações. Já no risco de lesão de pele foram registrados 7.179, com 17 eventos. E 3.446 pacientes/dia foram submetidos ao risco de extubação acidental, com 26 notificações. No risco de perda acidental de SNE e de SNG, foram registrados 7.034 pacientes/dia e 93 foram notificados. 7.550 pacientes/dia foram submetidos ao risco de perda acidental de cateter venoso central, e 18 eventos foram notificados. A taxa de efetividade em 2014 permaneceu acima de $98 \%$ nos riscos analisados, conforme exposto a seguir.

Tabela 1 - Número de pacientes submetidos ao risco/número de pacientes que desenvolveram o evento adverso de 2013. Caxias do Sul, RS, 2015

\begin{tabular}{lccccc} 
Mês & $\begin{array}{c}\text { Risco de } \\
\text { queda/ } \\
\text { Evento } \\
\text { adverso }\end{array}$ & $\begin{array}{c}\text { Risco de } \\
\text { I.pele/ } \\
\text { Evento } \\
\text { adverso }\end{array}$ & $\begin{array}{c}\text { Risco de extu. } \\
\text { aci./ } \\
\text { Evento } \\
\text { adverso }\end{array}$ & $\begin{array}{c}\text { Risco de } \\
\text { Sne Sng/ } \\
\text { Evento } \\
\text { adverso }\end{array}$ & $\begin{array}{c}\text { Risco de } \\
\text { cvc/ } \\
\text { Evento } \\
\text { adverso }\end{array}$ \\
\hline Janeiro & $0 / 0$ & $7 / 0$ & 3 -Jan & $14 / 0$ & $6 / 0$ \\
\hline Fevereiro & $104 / 1$ & $10 / 0$ & 5 -Feb & $15 / 0$ & 2 -Jan \\
\hline Março & $0 / 0$ & $7 / 0$ & 3 -Jan & $34 / 1$ & $0 / 0$ \\
\hline Abril & $18 / 0$ & $20 / 0$ & $10 / 0$ & $28 / 0$ & $16 / 0$ \\
\hline Maio & $725 / 0$ & $684 / 0$ & $279 / 0$ & $490 / 1$ & $634 / 0$ \\
\hline Junho & $643 / 1$ & $645 / 0$ & $211 / 1$ & $524 / 0$ & $589 / 0$ \\
\hline Julho & $602 / 0$ & $583 / 1$ & $234 / 4$ & $529 / 6$ & $541 / 2$ \\
\hline Agosto & $299 / 0$ & $302 / 2$ & $94 / 4$ & $247 / 3$ & $290 / 0$ \\
\hline Setembro & $554 / 0$ & $547 / 0$ & $184 / 2$ & $276 / 7$ & $548 / 2$ \\
\hline Outubro & $520 / 1$ & $511 / 1$ & $214 / 1$ & $388 / 5$ & $461 / 1$ \\
\hline Novembro & $253 / 0$ & $261 / 0$ & $51 / 0$ & $281 / 7$ & $200 / 0$ \\
\hline Dezembro & $842 / 0$ & $570 / 2$ & $240 / 2$ & $536 / 2$ & $501 / 0$ \\
\hline Taxa de efetividade & $99,88 \%$ & $99,88 \%$ & $90,37 \%$ & $98,98 \%$ & $99,95 \%$
\end{tabular}

L: Iesão; Extu: extubação; aci: acidental; Sne: sonda nasoentérica; Sng: Sonda nasogástrica; Cvc: cateter venoso central 
Tabela 2 - Número de pacientes submetidos ao risco/número de pacientes que desenvolveram o evento adverso de 2014. Caxias do Sul, RS, 2015

\begin{tabular}{lccccc} 
Mês & $\begin{array}{c}\text { Risco de } \\
\text { queda/ } \\
\text { Evento } \\
\text { adverso }\end{array}$ & $\begin{array}{c}\text { Risco de } \\
\text { I.pele/ } \\
\text { Evento } \\
\text { adverso }\end{array}$ & $\begin{array}{c}\text { Risco de extu. } \\
\text { aci./ } \\
\text { Evento } \\
\text { adverso }\end{array}$ & $\begin{array}{c}\text { Risco de } \\
\text { Sne Sng/ } \\
\text { Evento } \\
\text { adverso }\end{array}$ & $\begin{array}{c}\text { Risco de } \\
\text { cvc/ } \\
\text { Evento } \\
\text { adverso }\end{array}$ \\
\hline Janeiro & $791 / 1$ & $858 / 0$ & $297 / 3$ & $624 / 8$ & $596 / 3$ \\
\hline Fevereiro & $420 / 0$ & $833 / 0$ & $235 / 1$ & $769 / 4$ & $600 / 3$ \\
\hline Março & $821 / 0$ & $762 / 0$ & $285 / 1$ & $568 / 5$ & $712 / 1$ \\
\hline Abril & $500 / 3$ & $297 / 2$ & $153 / 1$ & $256 / 7$ & $337 / 4$ \\
\hline Maio & $596 / 0$ & $335 / 7$ & $335 / 3$ & $648 / 4$ & $670 / 1$ \\
\hline Junho & $849 / 0$ & $318 / 2$ & $309 / 4$ & $675 / 9$ & $724 / 0$ \\
\hline Julho & $851 / 0$ & $624 / 0$ & $315 / 5$ & $646 / 15$ & $700 / 2$ \\
\hline Agosto & $561 / 0$ & $648 / 3$ & $237 / 0$ & $416 / 2$ & $525 / 2$ \\
\hline Setembro & $791 / 0$ & $577 / 0$ & $318 / 3$ & $577 / 14$ & $661 / 1$ \\
\hline Outubro & $849 / 0$ & $596 / 1$ & $381 / 5$ & $671 / 4$ & $672 / 0$ \\
\hline Novembro & $813 / 0$ & $670 / 1$ & $321 / 0$ & $674 / 11$ & $658 / 0$ \\
\hline Dezembro & $784 / 0$ & $661 / 1$ & $260 / 0$ & $510 / 10$ & $695 / 1$ \\
\hline Taxa de efetividade & $99,93 \%$ & $99,79 \%$ & $99,28 \%$ & $98,59 \%$ & $99,70 \%$
\end{tabular}

L: lesão; Extu: extubação; aci: acidental; Sne: sonda nasoentérica; Sng: Sonda nasogástrica; Cvc: cateter venoso central

\section{DISCUSSÃO}

A cultura de segurança do paciente é essencial dentro de uma organização de saúde, pois é onde todos os trabalhadores, incluindo os profissionais envolvidos no cuidado e os gestores, assumem responsabilidade pela sua própria segurança, pela segurança de seus colegas, dos pacientes e de seus familiares ${ }^{(1)}$.

Os resultados deste estudo evidenciam que, possivelmente, com o passar do tempo, a cultura de segurança dos profissionais atuantes nas UTIs tornou-se uma prática, uma vez que percebe-se o aumento no número de registros de pacientes com risco e, consequentemente, a notificação dos eventos adversos.

As práticas de segurança do paciente tornaram-se importantes para as equipes que possivelmente entenderam que, para poder prevenir, é essencial identificar os principais riscos e eventos que ocorrem. Diante disto, é identificando a origem dos eventos que se propõem às medidas a serem tomadas para que esses eventos não ocorram novamente. Para este fim, foi criado o gerenciamento de risco, que é o caminho para se buscar um melhor controle e monitoramento de processos ${ }^{(11)}$.

Nas organizações com cultura de segurança instalada, os colaborados são tratados de forma justa e sem culpabilidade, para aprender com os erros, ao invés de culpar o indivíduo, fazendo com que a situação se transforme em uma abordagem sistematizada do problema. Essa cultura de segurança tem relação direta com o gerenciamento e com os valores da equipe, assim como os sentimentos e normas sobre o que priorizar na organização de cuidados à saúde, como devem se comportar os membros da equipe e quais processos devem ser revistos no que diz respeito à segurança do paciente ${ }^{(12)}$.

O conhecimento científico acerca deste tema deve iniciar nas UTIs, pois é um local que oferece maior risco à segurança do paciente, uma vez que é exigido um cuidado de alta complexidade, devido à gravidade dos pacientes e ao grande número de procedimentos e $\operatorname{processos}^{(12)}$.

Ao analisar os resultados, identificou-se que o risco de maior prevalência nos anos pesquisados foi o risco de perda acidental de SNE e SNG, somando um total de 125 eventos notificados. Já o gerenciamento de risco menos efetivo foi no risco de extubação acidental, com uma efetividade média 
de $94,82 \%$.

A extubação não planejada é uma preocupação frequente nas UTIs, que acomete de 3 a $22 \%$ dos pacientes. A extubação acidental é compreendida como a retirada prematura do dispositivo ventilatório pela ação do paciente, ou pela retirada não-intencional desse pelo profissional, mediante a manipulação do paciente. A extubação acidental ocorre devido à agitação psicomotora do paciente, inadequada fixação do dispositivo ventilatório, falta de adequada sedação, cuff do tubo orotraqueal furado ou vazio, tração ou peso excessivo dos acessórios do ventilador mecânico, bem como consequente ao manuseio inadequado do paciente pela equipe multiprofissional, caracterizando um evento adverso do cuidado(13).

Entretanto, há outros fatores relacionados à ocorrência deste evento nos pacientes submetidos à ventilação mecânica na UTI, como a inexperiência profissional, a quantidade insuficiente de pessoal, a falta de atenção e o desconhecimento técnico-científico dos integrantes da equipe multiprofissional e problemas inerentes aos materiais e equipamentos utilizados na assistência ao paciente grave ${ }^{(13)}$.

Quando ocorre a extubação, as consequências para o paciente podem ser diversas, pois sempre é necessária a reintubação e, com isso, há um aumento do tempo de ventilação mecânica, que gera maior tempo de internação e altos custos hospitalares, maior risco de hipoxemia, atelectasia, pneumonia associada à ventilação mecânica, trauma de vias aéreas, potencial instabilidade hemodinâmica, arritmias, parada cardiorrespiratória e cerebral ou mesmo a morte do paciente ${ }^{(13)}$.

Algumas barreiras utilizadas para a prevenção da extubação são: identificar o paciente precocemente com este risco potencial; se o paciente estiver orientado, esclarecer a necessidade do dispositivo; verificar a pressão do cuff a cada 12 horas; se o paciente estiver sedado, avaliar o nível de sedação, a fixação correta do tubo e a contenção motora em caso de agitação(14).

Diante do exposto, faz-se necessário que os profissionais de enfermagem saibam lidar com o paciente em ventilação mecânica. Ter conhecimento sobre essas tecnologias é essencial para proporcionar segurança ao paciente e prestar uma assistência de qualidade, tendo em vista que os cuidados de enfermagem buscam a prevenção de complicações associadas a pacientes que usam esse dispositivo. Este tema, apesar de ser bem difundido dentro dos cuidados intensivos e pelo fato de a extubação acidental ser considerada um importante indicador de qualidade do cuidado de enfermagem dentro da terapia intensiva, ainda há uma preocupação acerca da associação da extubação acidental aos cuidados prestados pela equipe de enfermagem ${ }^{(15)}$.

O enfermeiro, por meio da sistematização da assistência, pode assegurar ao paciente uma assistência adequada e individualizada que gere segurança e confiança ao enfermo e a sua família, diante de seus conhecimentos científicos. Entretanto, o que pode acontecer atualmente, é a existência de profissionais que atuam de forma assistemática, devido à resistência em mudar o seu cotidiano, tecnicista e fragmentado. No entanto, sugere-se que os enfermeiros se preocupem mais com a qualidade da assistência prestada, ao invés de limitar-se, predominantemente, às demandas do serviço, e que, de maneira precipitada, pode contribuir para o surgimento de extubações acidentais na UTI(15).

O evento de maior prevalência no estudo foi a perda acidental de SNE e de SNG, que é um risco muito comum nestas unidades. Estão expostos a estes riscos todos os pacientes que utilizam estes dispositivos. Algumas barreiras para evitar que esses incidentes ocorram são: fixação adequada; contenção motora em caso de agitação; cuidado no manuseio do paciente ${ }^{(16)}$.

Embora o uso da sonda enteral traga benefícios, também traz riscos e potencia eventos adversos, que podem ocorrer pela expulsão deliberada destes dispositivos pelo próprio paciente, que pode estar com agitação motora, uso de sedação, confusão mental, distúrbios neurológicos, entre outros. Os EAs podem também ocorrer durante os cuidados de enfermagem, como: na troca de curativos; na fixação inadequada da cânula; no banho ou higiene íntima; na administração de medicamentos; na mudança de decúbito; no transporte leito-maca; ou no transporte maca-leito ${ }^{(17)}$.

A enfermagem tem papel fundamental, pois atua na administração, manutenção e controle nutricional do paciente, e o profissional enfermeiro é responsável pela realização da sondagem, bem como sua manutenção(16). Diante disso, é essencial que a equipe de enfermagem realize os cuidados de enfermagem de forma organizada, a fim de assegurar maior segurança aos pacientes e, 
consequentemente, a diminuição deste evento.

Os erros e eventos adversos mostram-se frequentes no que tange à assistência à saúde. Em um estudo realizado, foi possível identificar que os profissionais recebem poucas informações acerca da ocorrência de erros e eventos adversos e sobre as mudanças implantadas em decorrência de falhas assistenciais. Ainda existe quantidade reduzida de notificações de erros e eventos adversos, bem como a maior parcela dos profissionais sinaliza que a instituição ainda apresenta postura punitiva às ocorrências ${ }^{(18)}$.

Diante disso, os resultados obtidos no presente estudo mostram que a taxa de efetividade permaneceu acima de $99 \%$ na maioria dos riscos pesquisados, e a taxa desses eventos se manteve abaixo de $10 \%$, considerando o número de pacientes-dia exposto. Esse resultado é reflexo de uma assistência segura e de qualidade, sem esquecer do gerenciamento dos riscos que acontece na instituição da pesquisa. Estudos mostram que a frequência de eventos em pacientes hospitalizados pode variar de 10 a $60 \%$ e, em pacientes de UTIs, $20 \%$. Portanto, comparando ao estudo realizado, a ocorrência de eventos está abaixo do que na maioria das instituições ${ }^{(19-20)}$.

É importante considerar a possível ocorrência de subnotificação de EAs. A notificação de eventos tem por objetivo diminuir a ocorrência desses, pois, diante das notificações e, consequentemente, da identificação dos principais eventos que ocorreram, pode-se criar medidas para a melhoria do cuidado ao paciente e evitar que as falhas se repitam e que erros semelhantes aconteçam. Alguns dos possíveis motivos de muitos desses eventos serem subnotificados são os profissionais de saúde temerem a punição, terem vergonha e a perda de prestígio $^{(18)}$.

Estes resultados evidenciam que os gestores estão cada vez mais preocupados com a segurança de seus pacientes e, com isso, estão estimulando suas equipes a vivenciar uma cultura de segurança, com treinamentos constantes. Como consequência, haverá a diminuição da ocorrência de eventos adversos e, com isso, diminuirão os custos da instituição, que poderá oferecer uma assistência mais segura e de melhor qualidade.

\section{- CONSIDERAÇÕES FINAIS}

Com a finalização desta pesquisa, nota-se que os objetivos propostos foram atingidos. No planejamento deste estudo, foi levantada a hipótese de que a taxa de efetividade nas UTIs seria maior que $90 \%$, o que se evidenciou nesta análise.

Conheceu-se que a taxa de efetividade dos cinco riscos pesquisados nos anos de 2013 e 2014, na maioria deles, permaneceu acima de 99\%. Diante disso, comparando a outros estudos, a ocorrência de EAs, na instituição pesquisada, está abaixo da maioria das instituições, considerando que, na maioria delas, os eventos em UTIs ocorrem em $20 \%$ dos pacientes e, neste estudo ocorrem em apenas $10 \%$, levando em consideração o número de pacientes/dia expostos aos mesmos.

O gerenciamento de risco menos efetivo, se comparado aos outros riscos, foi o risco de extubação acidental, com uma efetividade média de 94\%. A extubação acomete entre 3 e $22 \%$ dos pacientes. Constatou-se que a ocorrência destes eventos, dentro da terapia intensiva, é considerada grave, e que a enfermagem exerce papel fundamental na diminuição da sua ocorrência.

O evento que ocorreu com maior frequência foi o da perda acidental de SNE e de SNG, com a soma de 125 eventos dentro do período pesquisado, o que tornou possível comparar com a frequência em outros estudos. Através desta análise, foi possível considerar que provavelmente podem ter ocorrido subnotificações dos eventos pelos profissionais atuantes na instituição, visto que é voluntária.

Percebe-se também, que com o passar dos meses, possivelmente o conceito de cultura de segurança tornou-se mais conhecido e foi trabalhado com os profissionais, devido ao aumento de notificações de pacientes expostos ao risco e de notificações de EAs. Entre as limitações encontradas, teve-se o preenchimento inadequado dos formulários em alguns meses no ano de 2013, porém este fato não interferiu na análise dos dados, e também a escassez de estudos referentes a esta temática.

Contudo, vale ressaltar que o papel da enfermagem em relação ao tema abordado possui grande 
relevância, pois o enfermeiro é quem permanece maior tempo com o paciente e, com isso, tem condições de reconhecer o paciente com risco. É o enfermeiro quem propõe ações para que não ocorram EAs. Portanto, ele é um dos profissionais com maior potencial para o gerenciamento de riscos, mas é necessário que esses profissionais sejam capacitados para a identificação e a prevenção desses EAs. Da mesma forma, a equipe multiprofissional e a importância da notificação.

Sugere-se, então, novas publicações com relação a esta temática devido à escassez de estudos que avaliam a taxa de efetividade do gerenciamento de risco, com o intuito de subsidiar ações de redução de EAs e qualificar a assistência prestada.

\section{REFERÊNCIAS}

1. Ministério da Saúde (BR), Fundação Oswaldo Cruz (FIOCRUZ). Agência Nacional de Vigilância Sanitária (ANVISA). Documento de referência para o programa nacional de segurança do paciente. Fundação Oswaldo Cruz. Agência Nacional de vigilância Sanitária. 2014. [acesso em 16 abr 2016]. Disponível:

http://bvsms.saude.gov.br/bvs/publicacoes/documento_referencia_programa_nacional_seguranca.pdf

2. Gallotti RMD. Eventos adversos e óbitos hospitalares em serviços de emergências clínicas de um hospital universitário terciário: um olhar para a qualidade da atenção [doutorado]. São Paulo (SP): Faculdade de Medicina da Universidade de São Paulo; 2003.

3. Silva AEBC. Segurança do paciente: desafios para a prática e a investigação em Enfermagem. Rev. Eletr. Enfer. [Internet] 2010; 12(3) [acesso em 15 abr 2016]. Disponível: http://dx.doi.org/10.5216/ree.v12i3.11885

4. Batalha EMSS. Cultura de segurança do paciente na percepção de profissionais de enfermagem de uma instituição de ensino [dissertação]. São Paulo (SP): Faculdade de Medicina da Universidade de São Paulo; 2012.

5. Teixeira TCA. Análise da causa raiz de incidentes relacionados a segurança do paciente na assistência de enfermagem em unidades de internação, em um hospital privado, no interior de São Paulo [tese]. São Paulo (SP): Faculdade de Medicina da Universidade de São Paulo; 2012.

6. Fassini P, Hahn GV. Riscos à segurança do paciente em unidade de internação hospitalar: concepções da equipe de enfermagem. Rev. Enferm. UFSM. [Internet] 2012; 2(2) [acesso em 15 abr 2016]. Disponível:

http://dx.doi.org/10.5902/217976924966

7. Feldman LB. Acreditação de serviços de saúde e enfermagem. São Paulo: Grupo Editorial Bolina; 2009.

8. Lima HO, Dutra ECRO. Gerenciamento de riscos na saúde: aplicação na atenção hospitalar. Rev. Adm. hospitalar e Inovação em saúde. [Internet] 2010; 9(6) [acesso em 15 abr 2016]. Disponível:

http://revistas.face.ufmg.br/index.php/rahis/article/view/1114/828

9. Barbosa SFF, Mello JF. Cultura de segurança do paciente em terapia intensiva: recomendações da enfermagem. Texto Contexto Enfermagem. [Internet] 2013; 33(1124) [acesso em 16 abr 2016]. Disponível:

http://dx.doi.org/10.1590/S0104-07072013000400031

10. Forster AJ, Rose NGW, Walraven CV, Stiell L. Adverse events identified following an emergency department visit. Qual Saf Health Care. [Internet] 2008; 16(1) [acesso em 16 abr 2016]. Disponível:

https://dx.doi.org/10.1136/qshc.2005.017384

11. Hinrichsen SL, Oliveira CLF, Campos M, Possas L, Sabino G, Vilella AS. Gestão da Qualidade e dos riscos na segurança do paciente: estudo-piloto. RAHIS - Rev. Adm. Hospitalar e Inovação em Saúde. [Internet] 2011; 10(7) [acesso em 16 abr 2016]. Disponível: http://dx.doi.org/10.21450/rahis.v3i7.1400

12. Tomazoni A, Rocha PK, de Souza S, Anders JC, de Malfussi HFC. Cultura de segurança do paciente em unidades de terapia intensiva neonatal: perspectivas da equipe de enfermagem e médica. Rev. Latino-Am. Enfermagem. [Internet] 2014; 22(5) [acesso em 16 abr 2016]. Disponível : http://dx.doi.org/10.1590/0104-1169.3624.2477

13. Ramalho Neto JM, do Nascimento LB, da Silva GNS, de Menezes MS, da Nóbrega MML. Extubação acidental e os cuidados intensivos de enfermagem. Rev. Enferm. UFPE. [Internet] 2014; 8(11) [acesso em 16 abr 2016]. Disponível: https://dx.doi.org/10.5205/reuol.6679-58323-1-ED.0811201418 
14. Viana RAPP, Withaker IY. Enfermagem em terapia intensiva. Práticas e vivências. Porto Alegre: Artmed; 2011.

15. Silva LD, Castellões TMFW. Ações de enfermagem para a prevenção da extubação acidental. Rev. bras. enferm. [Internet] 2009; 62(4) [acesso em 16 abr 2016]. Disponível: http://dx.doi.org/10.1590/S0034-71672009000400008

16. Venturi KK. Qualidade do cuidado em UTI: relação entre o dimensionamento de pessoal de enfermagem e eventos adversos [dissertação]. Curitiba: Universidade Federal do Paraná; 2009.

17. Pereira SEM, Coelho MJ, de Mesquita AMF, Teixeira AO, Graciano SA. Causas da retirada não planejada da sonda de alimentação em terapia intensiva. Acta Paul. Enferm. [Internet] 2013; 26(4) [acesso em 16 abr 2016]. Disponível: http://dx.doi.org/10.1590/S0103-21002013000400007

18. de Souza VS, Kawamoto AM, de Oliveira JLC, Tonini NS, Fernandes LM, Nicola AL. Erros e Eventos Adversos: a interface com a cultura de segurança dos profissionais de saúde. Rev. Cogitare enferm. [Internet] 2015; 20(3) [acesso em 16 abr 2016]. Disponível: http://dx.doi.org/10.5380/ce.v20i3.40687

19. Novaretti MCZ, Santos EV, Quitério LM, Daud-gallotti. Sobrecarga de trabalho da Enfermagem e incidentes e eventos adversos em pacientes internados em UTI. Rev. bras. enferm. [Internet] 2014; 67(5) [acesso em 16 abr 2016]. Disponível: http://dx.doi.org/10.1590/0034-7167.2014670504

20. Rothschild JM, Landriganc P, Cronin JW, Kaushal R, Lockley SW, Burdick E.; et al. The critical care safety study: the incidence and nature of adverse events and serious medical errors in intensive care. Critic Care Med. [Internet] 2005; 33(8) [acesso em 16 abr 2016]. Disponível:

https://dx.doi.org/10.1097/01.CCM.0000171609.91035.BD 